\title{
Çift Pervaneli Çok Girişli Çok Çıkışı (TRMS) Sistemin Kontrolü için Ayrık Zaman PID Kontrolör Tasarımı ve Gerçek Zaman Uygulaması
}

\author{
${ }^{1}$ Ayhan Ozdemir, ${ }^{* 1}$ Ibrahim Mucuk and ${ }^{1}$ Selim Ilhan \\ ${ }^{* 1}$ Faculty of Engineering, Department of Electrical Electronics Engineering Sakarya University, Turkey
}

\section{Özet}

Günümüzde birçok ileri kontrolör tasarım yöntemi geliştirilmiş olmasına rağmen PID kontrolör basit yapısı, kolay uygulanabilirliği, bozuculara ve lineersizliklere karşı dayanıklılığı sayesinde yaygın olarak kullanılmaya devam etmektedir. Bu çalışmada helikopter simülatörü olarak kabul edilen çift pervaneli çok girişli çok çıkışlı (TRMS) sistemin kontrolü için ayrık zaman PID kontrolör tasarımı ve simülasyon/gerçek zaman sonuçları sunulmaktadır. TRMS modern hava araçlarındaki kontrol zorluklarını barındıran, kublaj etkisi bulunan, lineer olmayan sistemdir. Sistemde bulunan kublajlar bozucu olarak kabul edilmiş ve iki SISO sistem için iki adet ayrık zaman PID kontrolör tasarımı yapılmıştır. Simülasyon/Gerçek zaman sonuçları karşılaştırmalı olarak değerlendirilmiştir.

Anahtar Kelimeler: Ayrık Zaman PID, TRMS, Lineerleştirme, Modelleme

\begin{abstract}
Today, although many advanced controller design methods have been developed, PID controller continues to be widely used thanks to its simple structure, easy applicability, robustness to disturbances and nonlinearities. In this study, discrete time PID controller design, simulation and real-time experiment are presented for the control of twin rotor mimo system, which is considered as a helicopter simulator. TRMS is a non-linear system, which contains control difficulties in modern aircraft and has coupling effect. The coupling effect in the system are accepted as disturbance and two discrete time PID controllers are designed for two SISO systems. Simulation/Real-time results are evaluated comparatively.
\end{abstract}

Key Words: Discrete Time PID, TRMS, Linearization, Modelling

\section{Giriş}

TRMS sistemi helikopter ile benzer dinamik özelliklere sahip Feedback firması tarafindan geliştirilen bir deney setidir [1]. Şekil 1. ile gösterilen sistem, kule üzerindeki serbest bir çubuğun her iki ucuna birbirine dik olarak yerleştirilen pervaneler sayesinde, yatay ve dikey düzlemde hareket edebilmektedir. DC motorlar ile döndürülen pervanelerin üretmiş olduğu kuvvet ile sistemin yatay eksen ile yaptığı $\alpha_{v}$ açısı ve dikey eksenle yaptığı $\alpha_{h}$ açısı kontrol edilmektedir. Sistem nonlineer özelliktedir ve kublaj etkisi bulunmaktadır. TRMS sisteminde pervanelerin üretmiş olduğu kuvvet motorların dönme hızı değiştirilerek ayarlanmaktadır. Helikopterlerde ise bu kuvvet pervanelerin açıları değiştirilerek ayarlanmaktadır. Bu farklılıktan dolayı TRMS sisteminde bir helikoptere göre daha fazla kublaj etkisi vardır. TRMS'nin gerçek zaman kontrolü için PCL-812 I-O kartı bulunan bir bilgisayar kullanılmaktadır. TRMS Toolbox ve Real-time kernel(RTK) ile sistem MATLAB/Simulink tabanlı olarak kontrol edilmektedir [1].

TRMS sisteminin modelleme çalışmalarında üretici firma tarafından geliştirilen model sistem

*Corresponding author: Address: Faculty of Engineering, Department of Electrical Electronics Engineering Sakarya University, 54187, Sakarya TURKEY. E-mail address: ibrahimmucuk@ sakarya.edu.tr, Phone: +902642955825 
dinamiklerini karşılayamamaktadır. [2]' de yapılan çalışma da TRMS sistemine ait dinamik denklemler Newtoinan ve Lagrangian yaklaşımları ile elde edilmiştir. Bu çalışmada yapılan karşılaştırmada iki yöntemin yaklaşık olarak aynı doğrulukta olduğu görülmüştür. [3]'te TRMS sisteminin lineer zamanla değişen modeli sunulmuştur. [4]'te TRMS sistemine giriş olarak verilen artan frekansta işaret ve sistemin çıkış işaretleri kullanılarak sisteme ait lineer model elde edilmiştir.

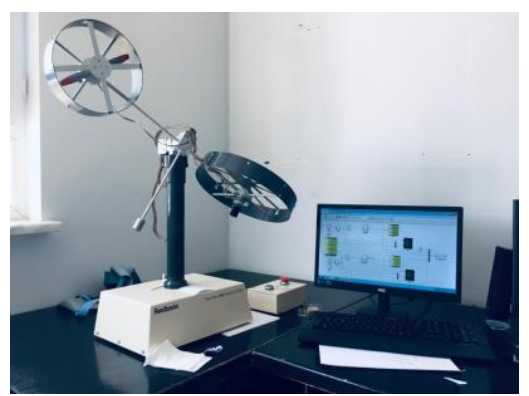

Şekil 1. TRMS gerçek görünümü

PID kontrolör basit yapısı ve uygulama kolaylığı ile kontrolör uygulamalarının çoğunda kullanılmaktadır. TRMS sisteminin kontrolü için PID kontrolör yapısının kullanıldığı birçok çalışma bulunmaktadır. [5]'te TRMS sistemi kontrolü için PID kontrolör katsayıları PSO tabanlı olarak bulunmuştur. [6]'da kesir dereceli PID kontrolör ile sistem kontrol edilmiş ve kontrolör katsayıları optimal olarak bulunmaktadır. [7], [8]'de bulanık mantık tabanlı PID kontrol ile TRMS sistemi kontrol edilmiştir. [9]'da TRMS sistemi kontrolünde PID ve IO-PID kontrolörleri performansı karşılaştırılmıştır. TRMS sistemi kontrolü için [10]'da ileri beslemeli ve geri beslemeli PID kontrolör uygulamaları gerçekleştirilmiştir. [5]-[7], [9] çalışmalarında bilgisayar gibi sayısal tabanlı kontrolör kullanılmasına rağmen kontrolör tasarımları sürekli zamanda yapılmış ve herhangi bir dönüşüm yapılmadan sayısal kontrolörlerde kullanılmıştır.

$\mathrm{Bu}$ çalışmada TRMS sistemine ait dinamik denklemler Newtonian yaklaşımı ile elde edilmiş ve modelde bilinmeyen parametreler gerçek zaman sistemden alınan giriş/çıkış verileri kullanılarak tahmin edilmiştir. Sistem belli bir çalışma bölgesinde lineerleştirilerek sisteme ait lineer model elde edilmiştir. Sistemde bulunan kublajlar bozucu kabul edilerek iki ayrı sayısal PID kontrolör tasarımı gerçekleştirilmiştir. Bölüm 2'de TRMS sisteminin matematik modeli elde edilmektedir. Kontrolör tasarım yöntemi bölüm 3'te verilmektedir. Bölüm 4'te simülasyon ve gerçek zaman çalışmanın sonuçları gösterilmektedir. Bölüm 5'te ise çalışmanın sonucu ve gelecek çalışmalar tartışılmaktadır.

\section{Sistemin Modellenmesi}

\subsection{TRMS Dinamik Denklemleri}

TRMS sistem modeli Şekil 2.'de gösterildiği gibi dikey düzlem ve yatay düzlem olmak üzere iki alt sisteme ayrılmıştır. Bu alt sistemler güç devreleri, DC (doğru akım:DA) motorlar, pervaneler ve yatay/dikey düzlemdeki mekanik aksamlardan oluşmaktadır. 
Sistemin giriş işaretleri olan $u_{1}$ ve $u_{2}$ işaretleri $-2.5 \mathrm{~V}$ ve $2.5 \mathrm{~V}$ arasında değişmekte ve motoru sürememektedir. Motorların sürülmesi için kullanılan güç devresinin uç denklemleri denklem 1 ile verilmiştir.

$V_{m / t}=u_{1 / 2} K_{1 / 2}$

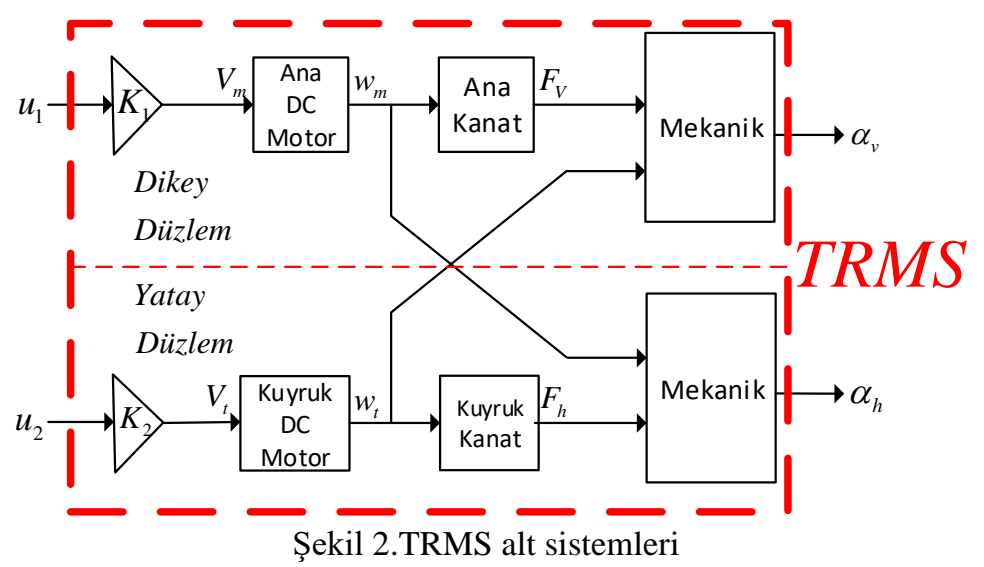

Sistemde kullanılan DC motorlarda armatür endüktansı sıfır alınarak alt sistem basitleştirilmiştir. DC motorların matematik modeli denklem 2 ile gösterilmektedir.

$J_{m v / t h} \frac{d w_{m / t}(t)}{d t}=K_{a v / a h} \frac{V_{m / t}(t)-K_{b v / t h} \omega_{m / t}(t)}{R_{a v / a h}}-B_{m v / t h} \omega_{m / t}(t)-T_{y m / y t}$

$T_{y t}= \begin{cases}k_{t v p / t h p} \omega_{m / t}\left|\omega_{m / t}\right| & \omega_{m / t} \geq 0 \\ k_{t v p / t h n} \omega_{m / t}\left|\omega_{m / t}\right| & \omega_{m / t}<0\end{cases}$

DC motorlar ile sürülen pervanelerin üretmiş oldukları kuvvetler denklem 3 ile verilmiştir.

$F_{v / h}= \begin{cases}k_{f v p / f h p} \omega_{m / t}\left|\omega_{m / t}\right| & \omega_{m / t} \geq 0 \\ k_{f v n / f h n} \omega_{m / t}\left|\omega_{m / t}\right| & \omega_{m / t}<0\end{cases}$

Sistemi oluşturan mekanik aksamların modeli Newton yaklaşımı ile elde edilmektedir. Sistemin dikey düzlemdeki hareketi denklem 4 ve denklem 5 ile verilmiştir. Denklem 4' te ilk terim yerçekimi kuvveti ile oluşan momenti, ikinci terim ana pervanenin oluşturmuş olduğu momenti, üçüncü terim hava sürtünmesi ile oluşan momenti, dördüncü terim merkez kaç kuvveti ile oluşan momenti, son terim ise jiroskop momentini ifade etmektedir. Denklem 5 'te $\frac{k_{t} \omega_{t}}{J_{v}}$ ifadesi sistemin yatay düzlemde hareketi sonucu oluşur ve açısal momentumum korunması kanunundan gelmektedir.

$\frac{d s_{v}}{d t}=\frac{g\left\{[A-B] \cos \alpha_{v}-C \sin \alpha_{v}\right\}+l_{m} F_{v}\left(\omega_{m}\right)-\Omega_{v} k_{v f v}-\Omega_{h}{ }^{2} H \cos \left(\alpha_{v}\right) \sin \left(\alpha_{v}\right)+k_{g} F_{v}\left(\omega_{m}\right) \Omega_{h} \cos \left(\alpha_{v}\right)}{J_{v}}$ 


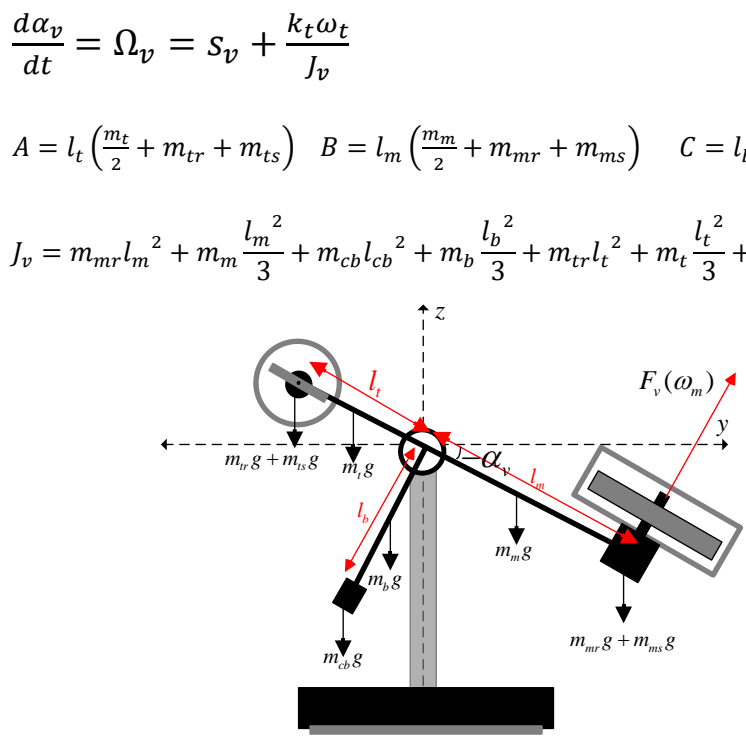

(a)

Şekil 3.(a) TRMS önden görünüşü (b) TRMS üstten görünüşü

Sistemin yatay düzlemdeki hareketi denklem 6 ve denklem 7 ile gösterilmiştir. Denklem 6' daki ilk terim kuyruk pervanesinin oluşturmuş olduğu momenti, ikinci terim hava sürtünmesi ile oluşan momenti, üçüncü ve dördüncü terimler kablo ağırlıklarının oluşturmuş olduğu momentleri ifade etmektedir. Denklem 7'deki $k_{m} \omega_{m} \cos \left(\alpha_{v}\right)$ ifadesi sistemin dikey düzlemde hareketi sonucu oluşur ve açısal momentumum korunması kanunundan gelmektedir.

$$
\begin{aligned}
& \frac{d s_{h}}{d t}=\frac{l_{t} F_{h}\left(\omega_{t}\right) \cos \left(\alpha_{v}\right)-\Omega_{h} k_{v f h}-M_{h 3}+M_{h 4}}{D \cos ^{2}\left(\alpha_{v}\right)+E \sin ^{2}\left(\alpha_{v}\right)+F} \\
& \frac{d \alpha_{h}}{d t}=\Omega_{h}=s_{h}+\frac{k_{m} \omega_{m} \cos \left(\alpha_{v}\right)}{D \cos ^{2}\left(\alpha_{v}\right)+E \sin ^{2}\left(\alpha_{v}\right)+F} \\
& D=m_{b} \frac{l_{b}{ }^{2}}{3}+m_{c b} l_{c b}{ }^{2} \quad E=\left(\frac{m_{m}}{3}+m_{m r}+m_{m s}\right) l_{m}{ }^{2}+\left(\frac{m_{t}}{3}+m_{t r}+m_{t s}\right) l_{t}{ }^{2} \quad F=m_{t s} \frac{r_{t s}{ }^{2}}{2}+m_{m s} r_{m s}{ }^{2} \\
& M_{h 3}=\left\{\begin{array}{lll}
k_{c h p} \alpha_{h} & \alpha_{h} \geq 0 \\
k_{c h n} \alpha_{h} & \alpha_{h}<0
\end{array} \quad M_{h 4}= \begin{cases}k_{c v p}\left(\alpha_{v}-\alpha_{v 0}\right)^{2} & \alpha_{v} \geq 0 \\
k_{c v n}\left(\alpha_{v}-\alpha_{v 0}\right)^{2} & \alpha_{v}<0\end{cases} \right.
\end{aligned}
$$

\subsection{Sistem Parametrelerinin Tahmini}

Denklem 1-7 ile verilen TRMS sistemine ait dinamik denklemler incelendiğinde hava sürtünmesi gibi birçok bilinmeyen/ölçülemeyen parametre olduğu görülmektedir. TRMS sistemi dinamik denklemlerinde bulunan bu parametreler gerçek zaman sistemden alınan giriş/çıkış verileri Şekil 4. ile gösterilen blok diyagram kullanılarak tahmin edilmiştir. 


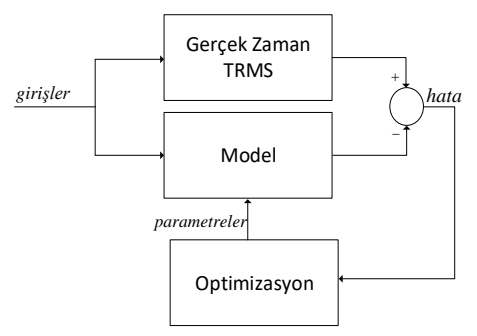

Şekil 4. Parametre tahmini blok diyagramı

TRMS sistem parametreleri Tablo 1. ile verilmiştir.

Tablo 1. TRMS sistem parametreleri

\begin{tabular}{|c|c|c|c|c|c|c|c|c|c|c|c|}
\hline Parametre & Değer & Parametre & Değer & Parametre & Değer & Parametre & Değer & Parametre & Değer & Parametre & Değer \\
\hline$l_{t}$ & 0,28494 & $m_{c b}$ & 0,0688 & $K_{a v}$ & 0,0202 & $k_{1}$ & 10 & $k_{f v p}$ & $2,0733 e-04$ & $k_{c v p}$ & 0,0623 \\
\hline$l_{m}$ & 0,24004 & $m_{t}$ & 0,0155 & $B_{m v}$ & $4,5 e-5$ & $k_{2}$ & 7,3837 & $k_{f v n}$ & $8,2682 \mathrm{e}-06$ & $k_{c v n}$ & 0,0025141 \\
\hline$l_{b}$ & 0,31 & $m_{b}$ & 0,022 & $K_{b v}$ & 0,0202 & $k_{t v p}$ & $\begin{array}{c}1,0073 e- \\
05\end{array}$ & $k_{f h p}$ & $1,3371 \mathrm{e}-04$ & $k_{c h n}$ & 0,039117 \\
\hline$l_{c b}$ & 0,3 & $m_{t s}$ & 0,1193 & $j_{t h}$ & $\begin{array}{c}2,5964 \mathrm{e}- \\
04\end{array}$ & $k_{t v n}$ & $5,1 \mathrm{e}-07$ & $k_{f h n}$ & $2,204 \mathrm{e}-06$ & & \\
\hline$r_{m s}$ & 0,155 & $m_{m s}$ & 0,2187 & $R_{a h}$ & 8 & $k_{t h p}$ & $\begin{array}{c}7,3726 \mathrm{e}- \\
06\end{array}$ & $k_{m}$ & $4,6316 e-04$ & & \\
\hline$r_{t s}$ & 0,1 & $g$ & 9,81 & $K_{a h}$ & 0,0202 & $k_{t h n}$ & $4,2 e-08$ & $k_{t}$ & 0,00035362 & & \\
\hline$m_{m r}$ & 0,2357 & $j_{m v}$ & $\begin{array}{c}5,8436 \mathrm{e}- \\
04\end{array}$ & $B_{t h}$ & $2,3 e-5$ & $k_{v f v}$ & 0,031427 & $k_{g}$ & $3,5793 e-04$ & & \\
\hline$m_{t r}$ & 0.2213 & $R_{a v}$ & 8 & $K_{b h}$ & 0,0202 & $k_{v f h}$ & 0,043986 & $k_{c h p}$ & 0,020237 & & \\
\hline
\end{tabular}

Aynı girişler için nonlineer dinamik denklemler ve tahmin edilen parametrelerle gerçekleştirilen simülasyon ve gerçek zaman sonuçların karşılaştırıldığı grafikler Şekil 5. ile gösterilmektedir.

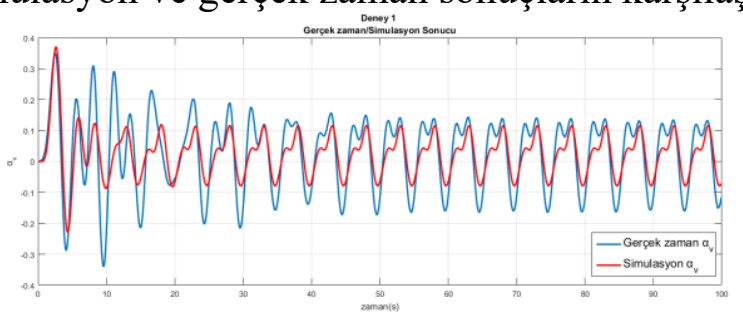

(a)

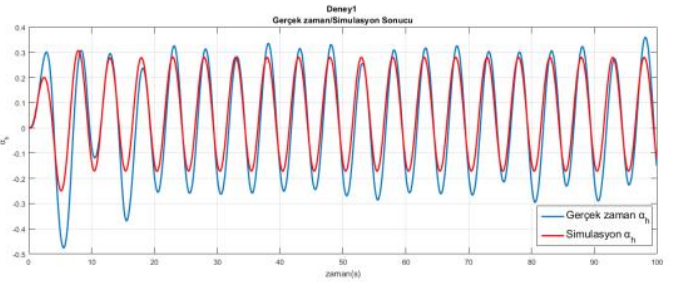

(b)

Şekil 5.(a) $\alpha_{v}$ simülasyon/gerçek zaman karşılaştırılması (b) $\alpha_{h}$ simülasyon/gerçek zaman karşılaştırılması

\subsection{Lineer Modelin Elde Edilmesi}

TRMS sisteminin lineer olmayan dinamik denklemleri belirli bir çalışma noktası etrafında Taylor serisine açılır ve 1 . Mertebeden yüksek terimleri atılır ise sisteme ait lineer denklemler denklem 8 
ile ifade edilir.

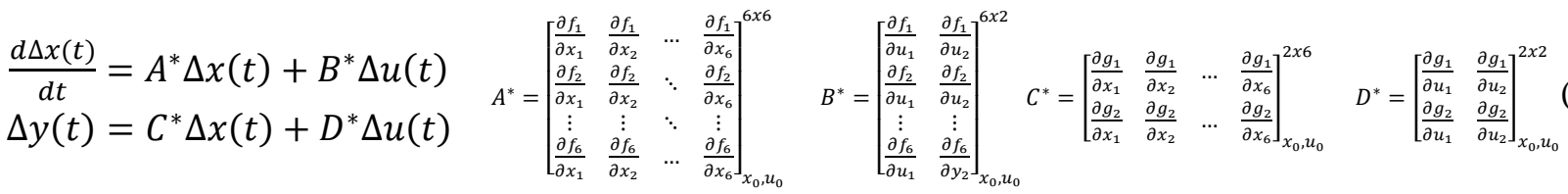

$$
\begin{aligned}
& \frac{d w_{m}}{d t}=43.2097 u_{1}-0.1642 w_{m}-0.01723 \omega_{m}^{2} \\
& \frac{d s_{v}}{d t}=0.000763 \omega_{m}^{2}-0.0026 \omega_{t}-1.7276 \cos \left(\alpha_{v}\right)-3.6055 \sin \left(\alpha_{v}\right)-0.4808 s_{v}-0.3644 \sin \left(2 \alpha_{v}\right)\left(s_{h}+\right. \\
& \left.\frac{0.00046 \omega_{m} \cos \left(\alpha_{v}\right)}{0.0545 \cos ^{2}\left(\alpha_{v}\right)+0.0069 \sin ^{2}\left(\alpha_{v}\right)+0.0059}\right)^{2}+0.0000011 \omega_{m}^{2} \cos \left(\alpha_{v}\right)\left(s_{h}+\frac{0.00046 w_{m} \cos \left(\alpha_{v}\right)}{0.0545 \cos ^{2}\left(\alpha_{v}\right)+0.0069 \sin ^{2}\left(\alpha_{v}\right)+0.0059}\right) \\
& \frac{d \alpha_{v}}{d t}=s_{v}+0.005415 \omega_{t} \\
& \frac{d w_{t}}{d t}=71.80651 u_{2}-0.2850 w_{t}-0.02839 \omega_{t}^{2} \\
& \frac{d s_{h}}{d t}=\frac{0.0202 \alpha_{h}+0.044 s_{h}-0.0623\left(\alpha_{v}+0.4479\right)^{2}-0.000038 \omega_{t}^{2} \cos \left(\alpha_{v}\right)+0.00046 w_{m} \cos \left(\alpha_{v}\right)}{0.0545 \cos ^{2}\left(\alpha_{v}\right)+0.0069 \sin ^{2}\left(\alpha_{v}\right)+0.0059} \\
& \frac{d \alpha_{h}}{d t}=s_{h}+\frac{0.0004631 \omega_{m} \cos \left(\alpha_{v}\right)}{D 0.0545 \cos ^{2}\left(\alpha_{v}\right)+0.0069 \sin ^{2}\left(\alpha_{v}\right)+0.0059}
\end{aligned}
$$

Denklem 9 ile verilen TRMS sistemi lineer olmayan model

$$
x_{0}=\left[\begin{array}{llllll}
41.6393 & -0.1575 & -0.1119 & 29.0861 & -0.3205 & 1.5967
\end{array}\right] u_{0}=\left[\begin{array}{ll}
0.85 & 0.45
\end{array}\right]
$$
çalışma noktası etrafındaki lineer durum uzay modeli denklem 10 ile verilmiştir.

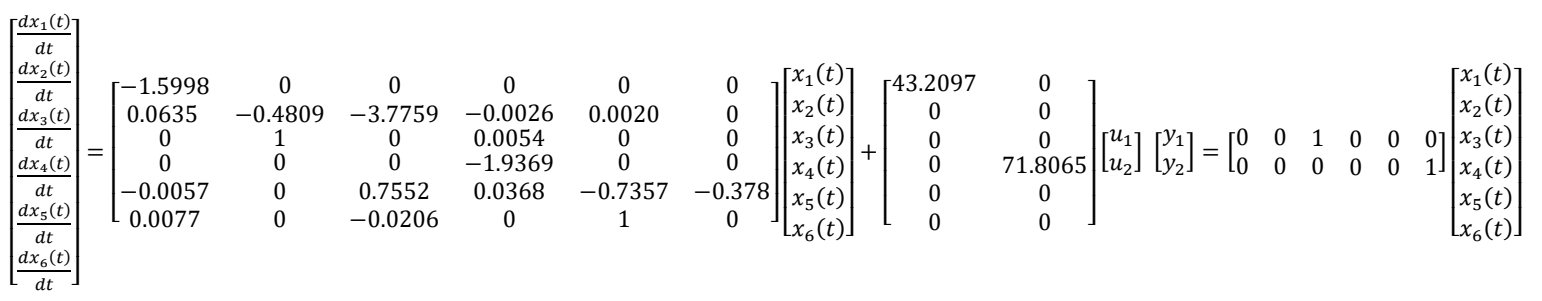

TRMS sistemine ait transfer fonksiyonu matrisi denklem 11'de gösterilmektedir ve Şekil 6.'da lineer transfer fonksiyonları ile TRMS sistemi blok diyagramı verilmiştir.

$\left[\begin{array}{l}y_{1} \\ y_{2}\end{array}\right]=\left[\begin{array}{ll}G_{p 1}(s) & G_{p 2}(s) \\ G_{p 3}(s) & G_{p 4}(s)\end{array}\right]\left[\begin{array}{l}u_{1} \\ u_{2}\end{array}\right]$

$G_{p 1}(s)=\frac{2.743 s^{2}+2.017 s+0.9261}{s^{5}+2.81 s^{4}+6.41 s^{3}+10.09 s^{2}+5.977 s+2.04}$

$G_{p 2}(s)=\frac{0.9889 s^{3}+0.2861 s^{2}+0.1365 s}{s^{5}+3.15 s^{4}+6.824 s^{3}+11.59 s^{2}+6.968 s+2.47}$

$G_{p 3}(s)=\frac{0.3325 s^{3}+0.1599 s^{2}+1.199 s+2.029}{s^{5}+2.81 s^{4}+6.41 s^{3}+10.09 s^{2}+5.977 s+2.04}$

$G_{p 4}(s)=\frac{2.636 s^{2}+1.559 s+9.983}{s^{5}+3.15 s^{4}+6.824 s^{3}+11.59 s^{2}+6.968 s+2.47}$ 


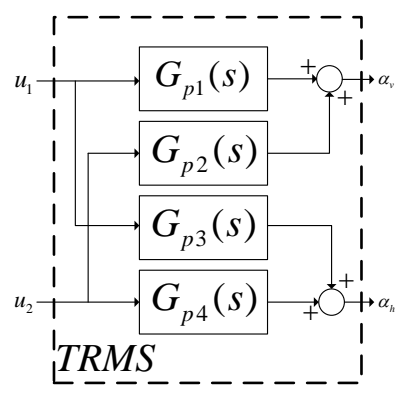

Şekil 6. Lineer transfer fonksiyonları ile TRMS sistemi

\section{PID Kontrolör Tasarımı}

[11]'te detaylı olarak anlatılan parametrik denklemler ile tasarım yöntemi sayısal PID kontrolör tasarımı kolaylaştırmaktadır. Sayısal PID kontrolör kapalı cevrim blok diyagramı Şekil 7.'de verilmektedir.

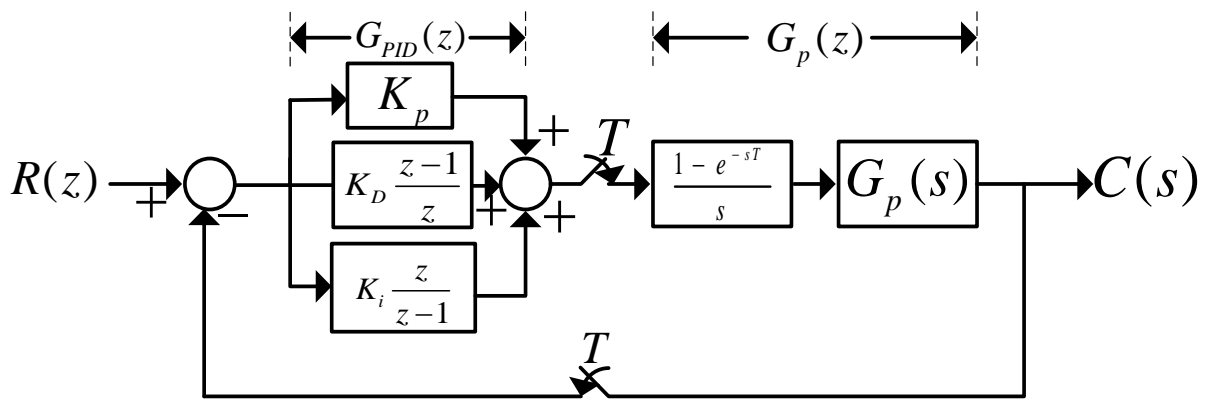

Şekil 7.Ayrık zaman PID kapalı çevrim kontrol blok diyagramı

Kontrolör tasarım aşamaları adım adım verilmiştir.

1.Sistem zaman sabitine göre örnekleme zamanı belirlenir ve sistemin ayrık zaman transfer fonksiyonu elde edilir.

$G_{p}(z)=\left.Z\left\{\frac{1-e^{-s T}}{s} G_{p}(s)\right\}\right|_{T}$

2. Kapalı çevrim sistemin performansı yüzde aşım $(\% M p)$ ve yerleşme zamanı $\left(t_{s}\right)$ seçilerek belirlenir. Sistemin doğal açısal frekansı $\left(w_{n}\right)$ ve sönüm oranı $(\xi)$ denklem 13 kullanılarak elde edilir.

$\% M p=e^{-\pi \frac{\xi^{2}}{\sqrt{1-\xi^{2}}}} \quad t_{s}=\frac{4}{\xi w_{n}} \% 2$ kriteriiçin

3. Hesaplanan doğal açısal frekans $\left(w_{n}\right)$ ve sönüm oranı $(\xi)$ denklem 14 'te yerine konularak sürekli ve ayrık zaman kontrol kökleri elde edilir. 
$s_{1,2}=-\xi w_{n} \pm j w_{n} \sqrt{1-\xi^{2}} \quad z_{1}=e^{s_{1} T}=\left|z_{1}\right| e^{j \beta}$

4. $z_{1}$ kompleks sayısı birinci aşamada elde edilen ayrık zaman transfer fonksiyonunda yerine konularak

$G_{p}\left(z_{1}\right)=\left|G_{p}\left(z_{1}\right)\right| e^{\psi}$

elde edilir.

5. Birim rampa giriş için sistemin sürekli hal hatası $e_{s s}=k_{h} \frac{2 \xi}{w_{n}}$ alınır ve

$\frac{1}{e_{s s}}=\frac{1}{T} \lim _{z \rightarrow 1}(z-1) G_{P I D}(z) G_{P}(z)$

formülü kullanılarak $K_{i}$ integratör katsayısı hesaplanır.

6. Hesaplanan $\left|z_{1}\right|, \beta,\left|G_{p}\left(z_{1}\right)\right|, \Psi$ ve $K_{i}$ değerleri denklem 17 ve 18 'de yerine konularak $K_{P}$ ve $K_{D}$ katsayıları hesaplanır.

$$
\begin{aligned}
\mathrm{K}_{\mathrm{p}} & =-\frac{\cos (\psi)}{\left|\mathrm{G}_{\mathrm{p}}\left(\mathrm{z}_{1}\right)\right|}-2 K_{i}\left|z_{1}\right| \frac{\left|z_{1}\right|-\cos (\beta)}{\left|z_{1}\right|^{2}-2\left|z_{1}\right| \cos (\beta)+1}+\frac{-\left|\mathrm{z}_{1}\right| \sin (\psi)+\cos (\beta) \sin (\psi)}{\left|\mathrm{G}_{\mathrm{p}}\left(\mathrm{z}_{1}\right)\right| \sin (\beta)} \\
K_{d} & =\frac{\left|z_{1}\right|}{\sin (\beta)}\left(\frac{K_{i} \sin (\beta)}{\left|z_{1}\right|-2 \cos (\beta)+\frac{1}{\left|z_{1}\right|}} \frac{\sin (\psi)}{\left|G_{p}\left(z_{1}\right)\right|}\right)
\end{aligned}
$$

\section{Simülasyon ve Gerçek Zaman Çalışma}

TRMS sisteminde $\alpha_{v}$ ve $\alpha_{h}$ çıkışları ayrık zaman PID kontrolör ile kontrol edilmiştir. Kontrolör tasarımında Denklem 11 ile verilen transfer fonksiyonu kullanılmıştır. Sistemde bulunan kublajlar bozucu kabul edilmiş ve $G_{p 1}(s)$ ve $G_{p 4}(s)$ transfer fonksiyonları için iki adet ayrık zaman PID kontrolör tasarımı gerçekleştirilmiştir. PID kontrolörlerin kullanıldığı kapalı çevrim bok diyagramı Şekil 8.'de gösterilmektedir.

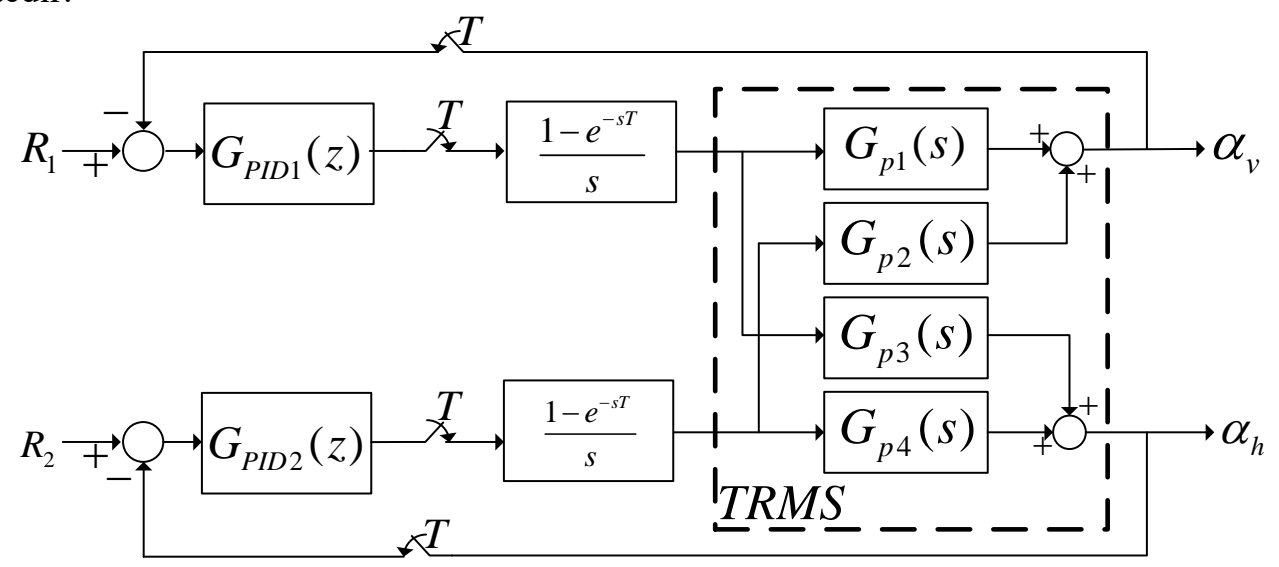

Şekil 8. TRMS ayrık zaman PID kontrolörlü kapalı çevrim kontrol blok diyagramı 
$G_{P I D 1}(z)$ tasarımında örnekleme zamanı $T=0.06$, performans kriteri olarak yüzde aşım $M_{p 1}=4$ yerleşme zamanı $t_{s 1}=6 s$ ve $k_{h 1}=1$ seçilmiştir. Denklem 12-18 kullanılarak $K_{P 1}=0.2022$ $K_{i 1}=0.0860 \quad K_{D 1}=10.4672$ olarak hesaplanmıştır.

$G_{P I D 2}(z)$ tasarımında örnekleme zamanı $T=0.06$, performans kriteri olarak yüzde aşım $M_{p 2}=4$ yerleşme zamanı $t_{s 2}=5 \mathrm{~s}$ ve $k_{h 2}=0.5$ olarak seçilmiştir. Denklem 12-18 kullanılarak $K_{P 2}=$ $0.7136 \quad K_{i 2}=0.0232 \quad K_{D 2}=12.1795$ olarak hesaplanmıştır.

Şekil 9.'da elde edilen modele ait kapalı çevrim simülasyon devresi ve gerçek zaman sistemde kullanılan Simulink ekran görüntüsü verilmektedir.

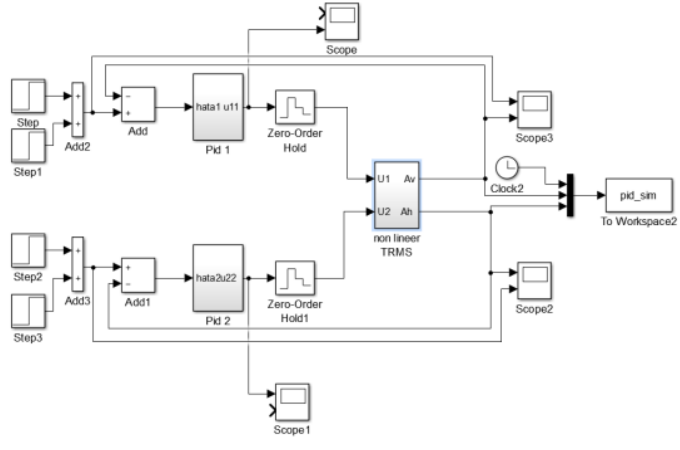

(a)

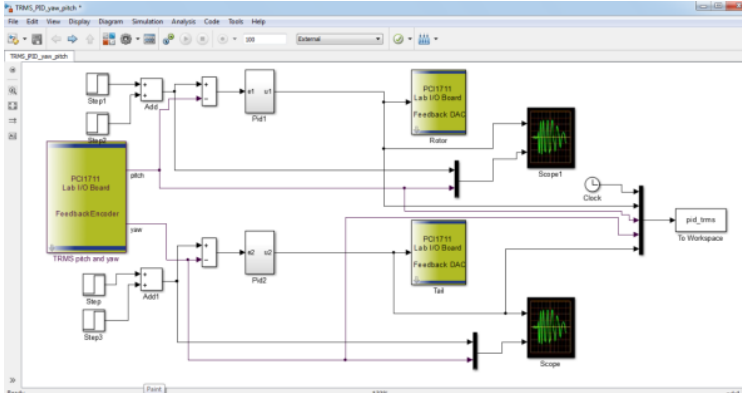

(b)

Şekil 9. (a) PID kontrolör simülasyonu (b) Gerçek zaman PID kontrolör

Tablo 2.'de parametrik denklemlere tasarlanan PID kontrolörlerin bulunduğu kapalı cevrim sistemler için uygulanan referans işaretler verilmektedir. Bu referans girişler için simülasyon ve gerçek zaman çıkışlar karşılaştırmalı Şekil 10-13. ile gösterilmektedir.

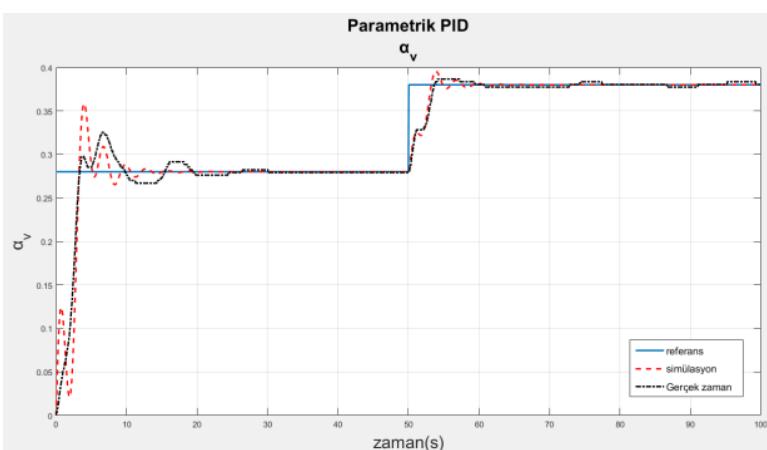

(a)

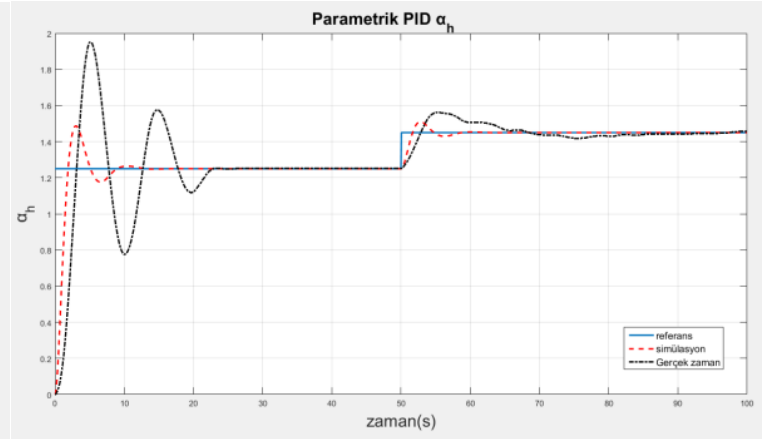

(b)

Şekil 10. a)Referans $1 \alpha_{v}$ çıkışı (b)Referans $1 \alpha_{h}$ çıkışı 


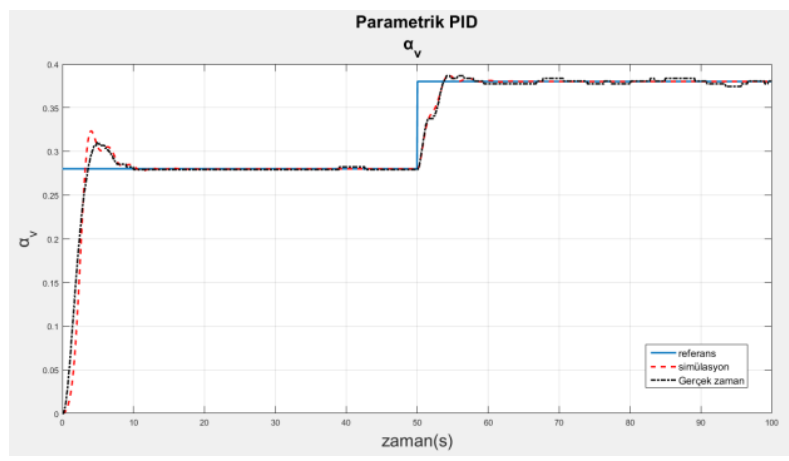

(a)

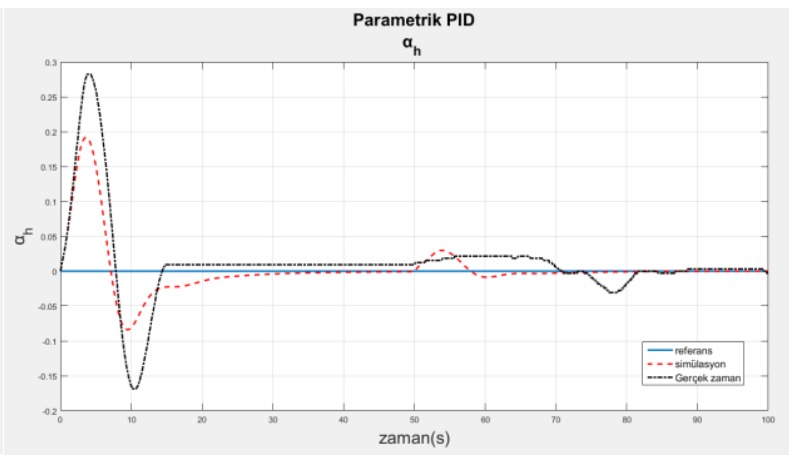

(b)

Şekil 11. (a)Referans $2 \alpha_{v}$ çıkışı (b)Referans $2 \alpha_{h}$ çıkış1

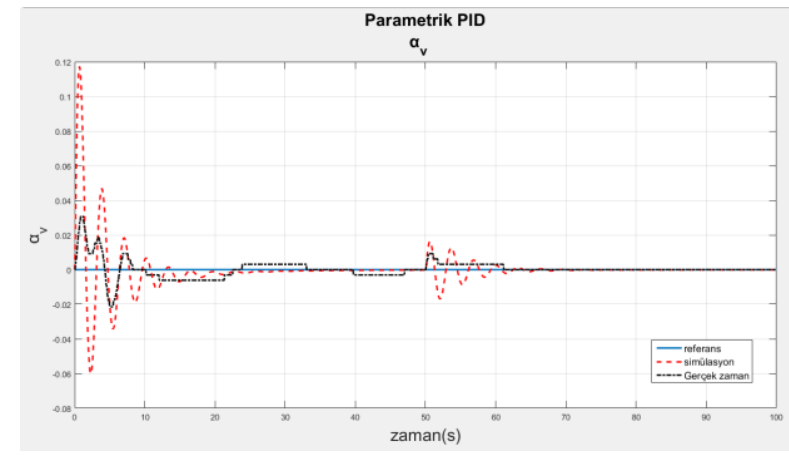

(a)

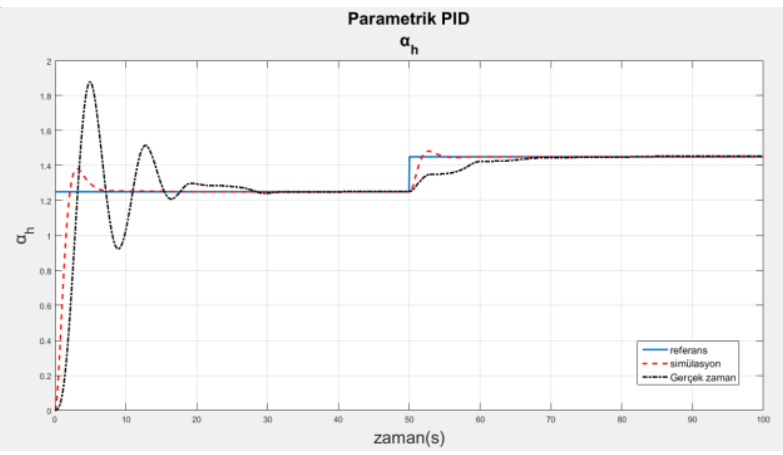

(b)

Şekil 12. (a)Referans $3 \alpha_{v}$ çıkışı (b)Referans $3 \alpha_{h}$ çıkışı

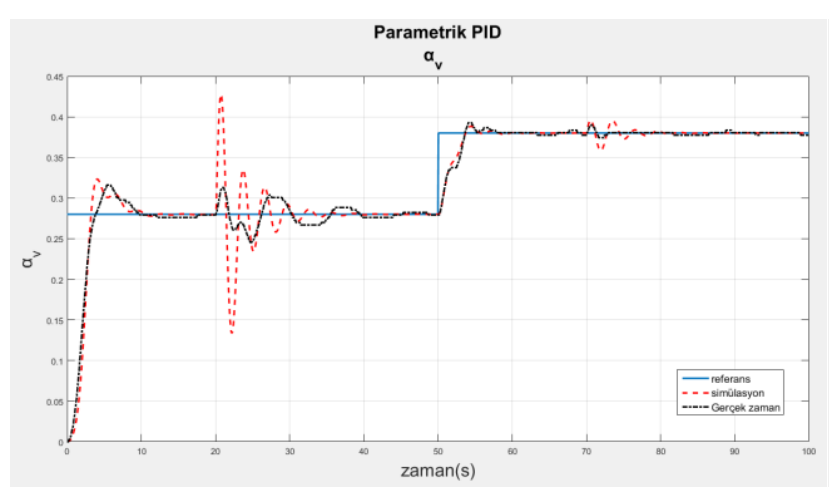

(a)

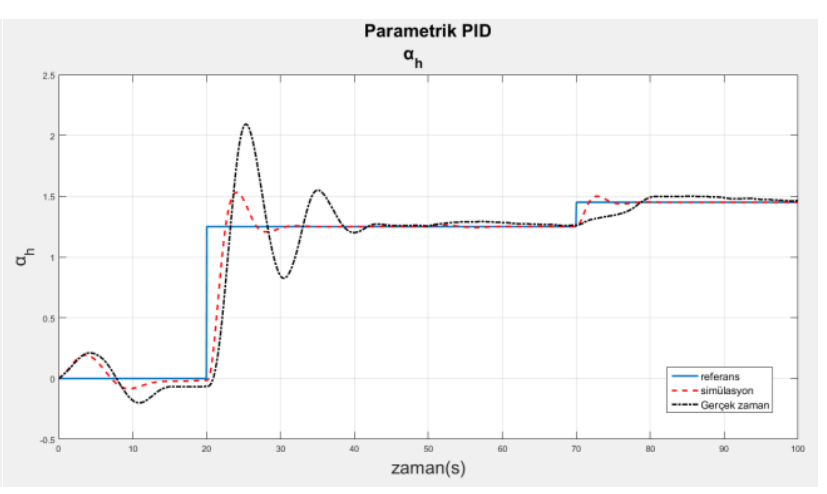

(b)

Şekil 13. (a)Referans $4 \alpha_{v}$ çıkışı (b)Referans $4 \alpha_{h}$ çıkışı 
Tablo 2. Uygulanan referans işaretler

\begin{tabular}{ccc}
\hline & & Giriş işareti \\
\hline \multirow{2}{*}{ Referans 1 } & $r_{1}$ & $0.28 \mathrm{u}(\mathrm{t})+0.1 \mathrm{u}(\mathrm{t}-50)$ \\
& $r_{2}$ & $1.25 \mathrm{u}(\mathrm{t})+0.2 \mathrm{u}(\mathrm{t}-50)$ \\
\hline \multirow{2}{*}{ Referans 2 } & $r_{1}$ & $0.28 \mathrm{u}(\mathrm{t})+0.1 \mathrm{u}(\mathrm{t}-50)$ \\
& $r_{2}$ & 0 \\
\hline \multirow{2}{*}{ Referans 3 } & $r_{1}$ & 0 \\
& $r_{2}$ & $1.25 \mathrm{u}(\mathrm{t})+0.2 \mathrm{u}(\mathrm{t}-50)$ \\
\hline \multirow{2}{*}{ Referans 4 } & $r_{1}$ & $0.28 \mathrm{u}(\mathrm{t})+0.1 \mathrm{u}(\mathrm{t}-50)$ \\
& $r_{2}$ & $1.25 \mathrm{u}(\mathrm{t}-20)+0.2 \mathrm{u}(\mathrm{t}-70)$ \\
\hline
\end{tabular}

\section{Sonuç}

TRMS sisteminin yatay ve dikey eksende açı kontrolü için tasarlanan PID kontrolör sonuçlarına bakıldığında PID kontrolörün lineer modelin elde edildiği çalışma bölgesinde belirlenen performansı sağladığ1 görülmektedir. Diğer çalışma bölgelerinde ise sistem referansı takip etmiş ancak belirlenen performansları sağlayamadığı gözlemlenmiştir. Tasarlanan kontrolörler her ne kadar kublaj etkilerini gidermiş olsalar da kublaj etkilerine hızlı cevap verememişlerdir. Tasarlanan kontrolörlerin performansını arttırmak için dekublatör tasarımı yapılabilir. Ayrıca farklı çalışma bölgelerinde sistemin lineer modeli elde edilip, her bölgede aynı performans kriterleri için PID katsayıları hesaplanırsa sistemin her bölgede aynı performansta çalışması sağlanabilir.

\section{Referanslar}

[1] C. Experiments, “Twin Rotor MIMO System Control Experiments," Work, vol. 44, no. 1160, 1998.

[2] A. Rahideh and M. H. Shaheed, "Mathematical dynamic modelling of a twin-rotor multiple input - multiple output system," vol. 221, pp. 89-101, 2007, doi:

10.1243/09596518JSCE292.

[3] D. Rotondo and F. Nejjari, "Control Engineering Practice Quasi-LPV modeling, identification and control of a twin rotor MIMO system," vol. 21, pp. 829-846, 2013, doi: 10.1016/j.conengprac.2013.02.004.

[4] P. K. Paul and J. Jacob, "ON THE MODELING OF TWIN ROTOR MIMO SYSTEM USING CHIRP INPUTS AS TEST SIGNALS,” vol. 19, no. 5, pp. 1731-1740, 2017, doi: 10.1002/asjc.1502.

[5] R. Maiti and K. Das Sharma, "PSO based parameter estimation and PID controller tuning for 2-DOF nonlinear twin rotor MIMO system PSO based parameter estimation and PID controller tuning for 2-DOF nonlinear twin rotor MIMO system Roshni Maiti *, Kaushik Das Sharma and Gautam Sarkar,” no. January, 2018, doi: 10.1504/IJAAC.2018.095109.

[6] O. W. Abdulwahhab and N. H. Abbas, "A New Method to Tune a Fractional-Order PID Controller for a Twin Rotor Aerodynamic System," Arab. J. Sci. Eng., vol. 42, no. 12, pp. 5179-5189, 2017, doi: 10.1007/s13369-017-2629-5. 
[7] J. Juang, W. Liu, and R. Lin, "A hybrid intelligent controller for a twin rotor MIMO system and its hardware implementation," ISA Trans., vol. 50, no. 4, pp. 609-619, 2011, doi: $10.1016 /$ j.isatra.2011.06.006.

[8] A. Rahideh and M. H. Shaheed, "Hybrid Fuzzy-PID-based Control of a Twin Rotor MIMO System," 2006.

[9] S. K. Valluru, "Design and Implementation of L-PID and IO-PID Controllers for Twin Rotor MIMO System," 2019.

[10] S. F. Toha and M. O. Tokhi, "PID and inverse-model-based control of a twin rotor system," vol. 29, pp. 929-938, 2011, doi: 10.1017/S0263574711000154.

[11] A. Ozdemir and Z. Erdem, "A new approach for calculation of PID parameters with model based compact form formulations," Elektron. ir Elektrotechnika, vol. 20, no. 3, pp. 3-10, 2014, doi: 10.5755/j01.eee.20.3.4415. 